\title{
Erratum to: QED in Krein Space Quantization
}

\author{
A. Zarei · B. Forghan · M.V. Takook
}

Published online: 27 September 2012

(C) Springer Science+Business Media, LLC 2012

\section{Erratum to: Int J Theor Phys (2011) 50:2466-2476 DOI 10.1007/s10773-011-0735-9}

The paper [A. Zarei et al., Int. J. Theor. Phys. 50 (2011) 2466] should be corrected by applying the following changes:

The formulae (3.8) and (3.22) which were mentioned in Sect. 3:

$$
\Pi_{k r}\left(k^{2}\right)=-\frac{e^{2}}{12 \pi^{2}} \ln \left(-\frac{k^{2}}{m^{2}}\right)-\frac{e^{2}}{6 \pi^{2}} \frac{k^{2}}{m^{2}}-\frac{e^{2}}{2 \pi^{2}} \int_{0}^{1} d x(1-x) x \ln \left(1-x(1-x) \frac{k^{2}}{m^{2}}\right),
$$

and

$$
F_{1}^{k r}\left(q^{2}\right)_{q^{2} \rightarrow 0}=-\frac{e^{2} q^{2}}{16 \pi^{2} m^{2}}+\frac{3 e^{2} q^{2}}{64 \pi^{2} m^{2}}-\frac{e^{2} q^{2}}{12 \pi^{2} m^{2}}\left(\ln \frac{m}{\mu}-\frac{3}{8}\right)
$$

should be corrected as follows:

$$
\begin{aligned}
\Pi_{k r}\left(k^{2}\right)= & \frac{e^{2}}{12 \pi^{2}} \ln \left(-\frac{k^{2}}{m^{2}}\right)+\frac{e^{2}}{2 \pi^{2}} \int_{0}^{1} d x(1-x) x \ln \left(1-x(1-x) \frac{k^{2}}{m^{2}}\right) \\
& -\frac{4 e^{2}}{\pi^{2}}\left\{-\frac{5}{36}-\frac{m^{2}}{3 k^{2}}+\frac{m^{4}}{3 k^{4}}+\left(\frac{1}{6}-\frac{m^{4}}{2 k^{4}}+\frac{m^{6}}{3 k^{6}}\right) \ln \left(1-\frac{k^{2}}{m^{2}}\right)\right\},
\end{aligned}
$$

The online version of the original article can be found under doi:10.1007/s10773-011-0735-9.

\section{A. Zarei - M.V. Takook}

Department of Physics, Science and Research Branch, Islamic Azad University, Tehran, Iran

B. Forghan

Department of Physics, Islamic Azad University, Parand Branch, Parand, Iran

M.V. Takook ( $\varangle)$

Department of Physics, Razi University, Kermanshah, Iran e-mail: takook@razi.ac.ir 


$$
F_{1}^{k r}\left(q^{2}\right)_{q^{2} \rightarrow 0}=\frac{\alpha q^{2}}{3 \pi m^{2}}\left(\ln \frac{m}{M}-\frac{3}{8}-\frac{1}{4}\right) .
$$

It is important to note that other equations and the conclusion in this paper are thoroughly correct and have not been affected by the above mentioned error. 